\title{
Phase-Type Models of Channel-Holding Times in Cellular Communication Systems
}

\author{
Christensen, Thomas Kaare; Nielsen, Bo Friis; Iversen, Villy Bæk
}

Published in:

I E E E Transactions on Vehicular Technology

Link to article, DOI:

10.1109/TVT.2004.825803

Publication date:

2004

Document Version

Publisher's PDF, also known as Version of record

Link back to DTU Orbit

Citation (APA):

Christensen, T. K., Nielsen, B. F., \& Iversen, V. B. (2004). Phase-Type Models of Channel-Holding Times in Cellular Communication Systems. I E E E Transactions on Vehicular Technology, 53(3), 725-733.

https://doi.org/10.1109/TVT.2004.825803

\section{General rights}

Copyright and moral rights for the publications made accessible in the public portal are retained by the authors and/or other copyright owners and it is a condition of accessing publications that users recognise and abide by the legal requirements associated with these rights.

- Users may download and print one copy of any publication from the public portal for the purpose of private study or research.

- You may not further distribute the material or use it for any profit-making activity or commercial gain

- You may freely distribute the URL identifying the publication in the public portal 


\title{
Phase-Type Models of Channel-Holding Times in Cellular Communication Systems
}

\author{
Thomas Kaare Christensen, Student Member, IEEE, Bo Friis Nielsen, Member, IEEE, and \\ Villy Bæk Iversen, Member, IEEE
}

\begin{abstract}
In this paper, we derive the distribution of the channel-holding time when both cell-residence and call-holding times are phase-type distributed. Furthermore, the distribution of the number of handovers, the conditional channel-holding time distributions, and the channel-holding time when cell residence times are correlated are derived. All distributions are of phase type, making them very general and flexible. The channel-holding times are of importance in performance evaluation and simulation of cellular mobile communication systems.
\end{abstract}

Index Terms-Call-holding time, cell-residence time, mobility.

\section{INTRODUCTION}

$\mathbf{P}$ ERFORMANCE analysis of cellular mobile communication systems requires a revision of the models used for classical telephone systems. The limiting resource is radio channels. In cellular mobile systems, a call may, by handover, change radio channel during the call-holding time. There are several reasons for this. The mobility of users may require a handover to a neighboring cell. Also, propagation conditions may change due to other subscribers or the environment and require handover to another channel (either in the same cell or a neighboring cell). The mobile system has high processing capabilities and it is possible to implement advanced strategies for resource allocation, e.g., in micro/macro cell systems or by exploiting the overlap area between cells, allowing a user to choose among different cells with acceptable transmission quality.

The channels of a cell are thus loaded by a mix of calls: new calls initiated inside the cell and handover calls from neighboring cells. Therefore, we need to distinguish between cell-residence time, the time a user is connected to the system (control or traffic channel) independent of whether there is a call or not; channel-holding time of a specific traffic channel; and call-holding time, which is all channel-holding times of a session added together.

Many of the classic teletraffic models are insensitive to the holding-time distribution, only the mean value being relevant for the blocking probability. This is the case for all state-dependent Poisson arrival processes [9]. We may assume that new

Manuscript received January 22, 2002; revised August 21, 2002 and March 3, 2003.

T. K. Christensen, deceased, was with Informatics and Mathematical Modeling, Technical University of Denmark, Lyngby 2800, Denmark.

B. F. Nielsen is with Informatics and Mathematical Modeling, Technical University of Denmark, Lyngby 2800, Denmark (e-mail: bfn@imm.dtu.dk).

V. B. Iversen is with the Center for Communications, Optics, and Materials, Technical University of Denmark, Lyngby 2800, Denmark (e-mail: vbi@com. dtu.dk).

Digital Object Identifier 10.1109/TVT.2004.825803 calls arrive according to a Poisson model, but the arrival process of handover calls are in general non-Poisson [13]. Thus, the channel-holding time distribution becomes important.

Also, for simulation studies, it is important to know the channel-holding time distribution to eliminate border effects when simulating systems with many cells.

Many papers have studied the channel-holding time distribution. Some consider the geometry of the cells and derive the channel-holding time distribution by assuming uniformly distributed users, directions, and speed. However, in the real world, cells are irregular in size and shape and the users are distributed according to buildings, roads, etc. Furthermore, a handover does not always happen because the mobile station moves out of range of the base station. Handovers to a new cell may be because of poor reception quality, which depends on things such as possible obstacles and other interfering signals. Traffic congestion can also result in a handover. It may thus be more appropriate to use probabilistic models, where distributions are obtained by field observations.

Early papers (e.g., [7]) concluded that, for most practical cases, both the channel-holding and the cell-residence times were well approximated by negative exponential distributions. More recent measurements [2], [3], however, show that this is not fulfilled today, where cells are smaller and the handover rate is higher. For Danish GSM1800 operators, there is, on average, one handover per call. The increase in data traffic with the introduction of new technologies, such as GPRS, Edge, and UMTS, may also result in more general cell-residence time distributions, since data traffic often is more variable than predicted by the exponential distribution. Additionally, a mixture of ordinary voice and data calls may not be well approximated by the negative exponential distribution either.

In this paper, we derive the channel-holding time for phasetype distributed call-holding and cell-residence times. This provides a model that is non-Poissonian but still analytically tractable and which easily can handle mixtures of different kinds of traffic. To model correlated cell-residence times, a Markovian arrival process (MAP) is used. This could be used to model different speeds of customers. The channel-holding time distribution has also been considered by Orlik and Rappaport [12] for sum of hyperexponential (SOHYP) distributions and by Fang and Chlamtac [5] for hyper-Erlang distributions, all of which are included in the phase-type distribution. We use the terminology shown in Fig. 1.

Forced handover due to, e.g., interference from channels in the same cell can be included by keeping the same cell-residence time, but decomposing the channel-holding time into a 


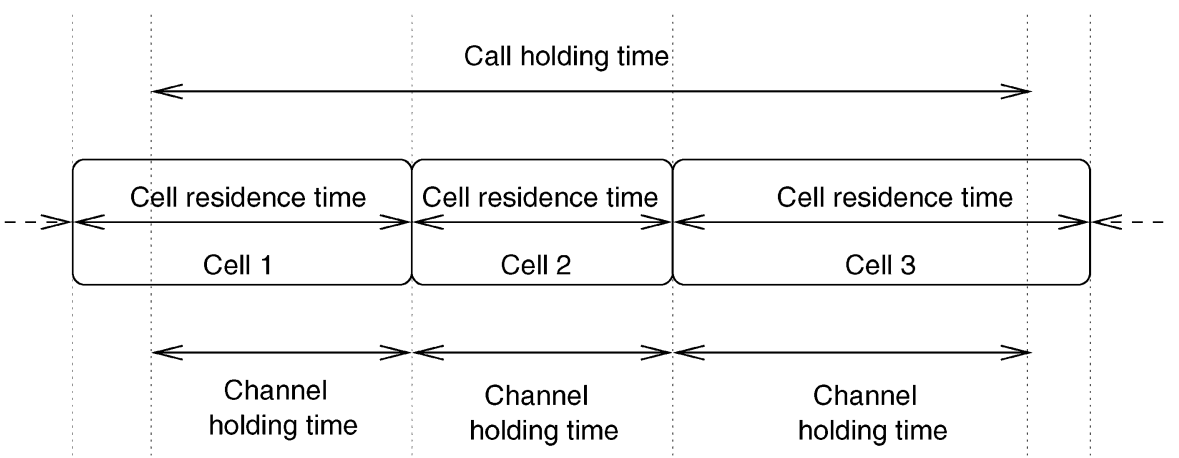

Fig. 1. Terminology applied in this paper. The call-holding time is the unencumbered call (session) holding time. The cell-residence time is the time interval a user spends within a cell (independent of whether there is a call or not). The channel-holding time is the time interval that a radio channel in a cell is occupied by a call.

stochastic number of subchannel-holding times. The simplest model will assume forced handover according to a Poisson process, but more complex models are feasible at the cost of increased complexity.

The physical cell structure such as sectorized cells has no influence on the models, which are based on cell-residence times. Forced handover to overlapping neighboring cells due to interference or high traffic load can be included by modifying the cell-residence time distribution.

This paper assumes that call-holding times are independent, which is only valid for a congestion-free system. It is not possible to include load dependent handover probabilities or to calculate congestion probabilities. The aggregated arrival process becomes very complex and can only be modeled by making further assumptions [13].

Phase-type models are very tractable for performance evaluation. Building on Markov chain theory systems can be analyzed by standard techniques in teletraffic and queueing theory. Recent examples in mobile communications are Litjens and Boucherie [11] and Christensen [4].

This paper significantly generalizes and enhances previous work [5], [6], [12], [15] on channel-holding times. It contains a very short introduction to phase-type distributions in Section II-A and then goes on to describe the derivation of the transition matrix between handovers in Section II-B, which is essential to the following derivations. Using this result, the distribution of the number of cells visited and channel-holding times are derived in Sections II-C and D, respectively. This is extended with conditional channel-holding times in Section II-E. Using the framework established in the first part of the paper, a final model incorporating correlation between cell-residence times is derived in Section II-F. Finally, a case study is presented in Section III, giving an example of the application of the model, and concluding remarks are given in Section IV.

\section{MODEL OF CHANNEL-HoLDING TIMES}

The phase-type model of channel-holding times is constructed in a stepwise fashion. Considering the evolution of the call-holding time from handover to handover leads to an explicit expression for the distribution of the channel-holding time. Based on this, channel-holding time distributions conditioned on termination of channel occupancy due to call termination and handover are found. Finally, the model is extended to include correlated cell-residence times by assuming that handovers occur according to a MAP.

\section{A. Phase-Type Distribution}

The phase-type distribution is defined as the time until absorption in a Markov chain with $m$ transient states and one absorbing state. It is parameterized by $(\underline{\alpha}, \boldsymbol{T})$, where $\underline{\alpha}$ is the probability distribution of the phases at time zero, $\alpha_{m+1}$ is the probability of immediate absorption, and $\boldsymbol{T}$ is the transient part of the generator matrix. The generator matrix for the entire Markov chain is

$$
\left[\begin{array}{cc}
\boldsymbol{T} & \underline{T}^{0} \\
\underline{0} & 0
\end{array}\right] .
$$

The vector $\underline{T}^{0}$ contains the intensities of absorption from the transient states. The probability density function (pdf) is

$$
f(x)=\underline{\alpha} e^{\boldsymbol{T} x} \underline{T}^{0} .
$$

In this paper, $\underline{e}$ is a column vector of a suitable dimension with all elements equal to one. An introduction to phase-type distributions is given in [10].

\section{B. Derivation of the Transition Matrix Between Handovers}

Let the stochastic variables $X_{0}, X_{i}$, and $Y$, respectively, describe the remaining cell-residence time in the first cell from the time a call is initiated, the cell-residence time after the $i$ th handover, and the remaining call-holding time in the last cell. Let the stochastic variable $N$ denote the number of handovers. Then, the call-holding time is $S=X_{0}+X_{1}+\cdots+X_{N-1}+Y$, for $N \geq 1$, and $S=Y$ otherwise. For the sake of simplicity, intracell handovers are not considered; however, they can easily be included in the model.

The distribution $S$ is assumed to be of continuous phase-type $S \in P H\left(\underline{\delta}_{0}, T\right)$ with $m$ states. The pdfs for $X_{0}, X_{i}$, and $S$ are denoted $g_{0}(t), g(t)$, and $f(t)$, respectively.

Let $\tau_{i}$ be the time instant of the $i$ th cell-residence time and let $J(t)$ be the phase of the remaining call-holding time distribution at time $t$. Define $\delta_{i, j}=P\left\{J\left(\tau_{i}\right)=j\right\}$ such that $\delta_{i, j}$ is the probability that the Markov chain at time $\tau_{i}$ is in phase $j$ or, 
in other words, the probability of being in phase $j$ at the $i$ th handover. The conditional probabilities are

$$
\left[P\left\{J\left(\tau_{1}\right)=1 \mid \tau_{1}=u\right\}, \ldots, P\left\{J\left(\tau_{1}\right)=m \mid \tau_{1}=u\right\}\right]=\underline{\delta}_{0} e^{\boldsymbol{T} u}
$$

implying that the unconditional probabilities are given by the compound distribution

$$
\begin{aligned}
\underline{\delta}_{1} & =\left(\delta_{1,1}, \ldots, \delta_{1, m}\right)=\underline{\delta}_{0} \int_{0}^{\infty} e^{\boldsymbol{T} u} g_{0}(u) d u \\
\underline{\delta}_{i} & =\left(\delta_{i, 1}, \ldots, \delta_{i, m}\right)=\underline{\delta}_{i-1} \int_{0}^{\infty} e^{\boldsymbol{T} u} g(u) d u, \quad i \geq 2 .
\end{aligned}
$$

The elements $\delta_{i, j}$ of $\underline{\delta}_{i}$ are the joint probabilities of having experienced $i$ handovers and being in phase $j$ at that handover. Thus, the elements of the vectors $\underline{\delta}_{i}$ will not sum to one.

We use uniformization [10] to rewrite the expression for $\underline{\delta}_{i}$. Let $\theta=\max \left\{\left|T_{i i}\right|: 1 \leq i \leq m\right\}$. $\theta$ is then the absolute value of the smallest element in the diagonal of $\boldsymbol{T}$, since all diagonal elements are negative. Define the uniformization matrix $\boldsymbol{A}=$ $\boldsymbol{I}+\theta^{-1} \boldsymbol{T}$; then, $\boldsymbol{T}=\theta(\boldsymbol{A}-\boldsymbol{I}) . A$ is now the transition matrix of a discrete Markov chain and $\theta$ is the intensity of the exponential distribution of time spent in each phase. The following property is used:

$$
e^{\boldsymbol{T} u}=e^{\theta(\boldsymbol{A}-\boldsymbol{I}) u}=e^{-\theta \boldsymbol{I} u} e^{\theta \boldsymbol{A} u}=e^{-\theta u} \sum_{i=0}^{\infty} \frac{(\theta \boldsymbol{A} u)^{i}}{i !} .
$$

Using (4) in the expression for $\underline{\delta}_{1}$, we get

$$
\begin{aligned}
\underline{\delta}_{1} & =\underline{\delta}_{0} \int_{0}^{\infty} e^{-\theta u} \sum_{i=0}^{\infty} \frac{(\theta \boldsymbol{A} u)^{i}}{i !} g_{0}(u) d u \\
& =\underline{\delta}_{0} \sum_{i=0}^{\infty} \frac{\boldsymbol{A}^{i}}{i !} \int_{0}^{\infty} e^{-\theta u}(\theta u)^{i} g_{0}(u) d u \\
& =\underline{\delta}_{0} \sum_{i=0}^{\infty} \frac{\boldsymbol{A}^{i}}{i !} E\left(e^{-\theta X_{0}}\left(\theta X_{0}\right)^{i}\right) \\
& =\underline{\delta}_{0} \boldsymbol{K}_{\mathbf{0}} .
\end{aligned}
$$

In a similar way, we get

$$
\underline{\delta}_{i}=\underline{\delta}_{i-1} K, \quad i \geq 2
$$

with

$$
\boldsymbol{K}=\sum_{j=0}^{\infty} \frac{A^{j}}{j !} E\left(e^{\theta X_{i}}\left(\theta X_{i}\right)^{j}\right) .
$$

Using (5) and the recursive formula (6), we get

$$
\underline{\delta}_{i}=\underline{\delta}_{0} \boldsymbol{K}_{\mathbf{0}} \boldsymbol{K}^{i-1}, \quad i \geq 1 .
$$

$K_{\mathbf{0}}$ and $K$ can be interpreted as transition matrices describing the transitions between phases from one handover to the next. The element $K_{i j}$ is the probability of being in phase $j$ at a handover, conditioned on being in phase $i$ at the previous handover. A transition to the absorbing state means that the call terminates in the cell. With the additional assumption that $X_{0}$ and $X_{i}$ are phase-type distributed with representations $\left(\underline{\beta}_{0}, \boldsymbol{S}_{\mathbf{0}}\right)$ with $n_{0}$ states, and $(\underline{\beta}, \boldsymbol{S})$ with $n$ states, $\boldsymbol{K}_{\mathbf{0}}$ and $\boldsymbol{K}$ are given by

$$
\begin{aligned}
\boldsymbol{K}_{\mathbf{0}} & =\sum_{i=0}^{\infty} \boldsymbol{A}^{i} \underline{\beta}_{0} \theta^{i}\left(\theta \boldsymbol{I}-\boldsymbol{S}_{\mathbf{0}}\right)^{-(i+1)} \underline{S}_{0}{ }^{0} \\
\boldsymbol{K} & =\sum_{i=0}^{\infty} \boldsymbol{A}^{i} \underline{\beta}^{i}(\theta \boldsymbol{I}-\boldsymbol{S})^{-(i+1)} \underline{S}^{0}
\end{aligned}
$$

since the exponentially weighted expectations are given by

$$
\begin{aligned}
E\left\{e^{-\theta X_{0}}\left(\theta X_{0}\right)^{j}\right\} & =j ! \underline{\beta}_{0} \theta^{j}\left(\theta \boldsymbol{I}-\boldsymbol{S}_{\mathbf{0}}\right)^{-(j+1)} \underline{S}_{0}{ }^{0} \\
E\left\{e^{-\theta X_{i}}\left(\theta X_{i}\right)^{j}\right\} & =j ! \underline{\beta} \theta^{j}(\theta \boldsymbol{I}-\boldsymbol{S})^{-(j+1)} \underline{S}^{0} .
\end{aligned}
$$

Alternatively, let $\gamma_{0}=\max \left\{\left|S_{0, i i}\right|: 1 \leq i \leq n_{0},\left|T_{i i}\right|: 1 \leq\right.$ $i \leq m\}$. Then, $\gamma_{0}$ is the absolute value of the smallest element in the diagonal of $S_{0}$ and $T$, since all diagonal elements are negative. Define the uniformization matrices $\boldsymbol{A}=\boldsymbol{I}+\gamma_{0}^{-1} \boldsymbol{T}$ and $\boldsymbol{B}_{\mathbf{0}}=\boldsymbol{I}+\gamma_{0}^{-1} \boldsymbol{S}_{\mathbf{0}}$. Then, $\boldsymbol{T}=\gamma_{0}(\boldsymbol{A}-\boldsymbol{I})$ and $\boldsymbol{S}_{\mathbf{0}}=\gamma_{0}\left(\boldsymbol{B}_{\mathbf{0}}-\boldsymbol{I}\right)$. The following property is used:

$$
g_{0}(u)=\underline{\beta}_{0} e^{\boldsymbol{S}_{\mathbf{0}} u} \underline{S}_{0}^{0}=\underline{\beta}_{0} e^{-\gamma_{0} u} \sum_{j=0}^{\infty} \frac{\left(\gamma_{0} \boldsymbol{B}_{\mathbf{0}} u\right)^{j}}{j !} .
$$

Then

$$
\begin{aligned}
\underline{\delta}_{1}= & \underline{\delta}_{0} \int_{0}^{\infty} e^{-\gamma_{0} u} \sum_{i=0}^{\infty} \frac{\left(\gamma_{0} \boldsymbol{A} u\right)^{i}}{i !} \underline{\beta}_{0} e^{-\gamma_{0} u} \\
& \times \sum_{j=0}^{\infty} \frac{\left(\gamma_{0} \boldsymbol{B}_{\mathbf{0}} u\right)^{j}}{j !} \underline{S}_{0}^{0} d u \\
= & \underline{\delta}_{0} \sum_{i=0}^{\infty} \sum_{j=0}^{\infty} \int_{0}^{\infty} e^{-2 \gamma_{0} u} \frac{\left(2 \gamma_{0} u\right)^{i+j}}{(i+j) !} 2 \gamma_{0} d u \\
& \times \frac{(i+j) !}{2^{i+j}} \frac{\boldsymbol{A}^{i}}{i !} \underline{\beta}_{0} \frac{\boldsymbol{B}_{0}^{j}}{j !} \frac{\underline{S}_{0}^{0}}{2 \gamma_{0}} \\
= & \underline{\delta}_{0} \sum_{i=0}^{\infty} \sum_{j=0}^{\infty} \frac{(i+j) !}{i ! j !}\left(\frac{\boldsymbol{A}}{2}\right)^{i} \underline{\beta}_{0}\left(\frac{\boldsymbol{B}_{\mathbf{0}}}{2}\right)^{j} \frac{\underline{S}_{0}^{0}}{2 \gamma_{0}} \\
= & \underline{\delta}_{0} \sum_{k=0}^{\infty} \sum_{l=0}^{k} \frac{(k) !}{l !(k-l) !}\left(\frac{\boldsymbol{A}}{2}\right)^{l} \underline{\beta}_{0}\left(\frac{\boldsymbol{B}_{\mathbf{0}}}{2}\right)^{k-l} \frac{\underline{S}_{0}^{0}}{2 \gamma_{0}} \\
= & \underline{\delta}_{0} K_{\mathbf{0}}
\end{aligned}
$$

and

$$
\boldsymbol{K}=\sum_{k=0}^{\infty} \sum_{l=0}^{k} \frac{k !}{l !(k-l) !}\left(\frac{\boldsymbol{A}}{2}\right)^{l} \underline{\beta}\left(\frac{B}{2}\right)^{k-l} \underline{\underline{S}}^{0} .
$$

The advantage of calculating $K_{\mathbf{0}}$ and $K$ in this manner is that matrix inversion is no longer needed.

\section{Distribution of the Number of Cells Visited}

Let the stochastic variable $N$ denote the number of handovers. The probability of no handover is equivalent to the probability of absorption in the underlying Markov chain of the call-holding time before time instant $\tau_{1}$ or, in other words, the probability of absorption before the first handover

$$
p_{0}=P\{N=0\}=1-\underline{\delta}_{1} \cdot \underline{e}=1-\underline{\delta}_{0} \boldsymbol{K}_{\mathbf{0}} \underline{\underline{e}} .
$$


The probability of at least $i$ handovers, which is the same as the probability that the call survives the $i$ th cell, is given by

$$
q_{i}=P\{N \geq i\}=\underline{\delta}_{i} \cdot \underline{e}=\underline{\delta}_{0} \boldsymbol{K}_{\mathbf{0}} \boldsymbol{K}^{i-1} \underline{e}, \quad i \geq 1 .
$$

Obviously, $q_{0}=\underline{\delta}_{0} \underline{e}=1$. The distribution of the number of handovers is then a discrete phase-type distribution

$$
\operatorname{PH}\left(\left(\underline{\delta}_{0}, \underline{0}\right),\left[\begin{array}{cc}
\mathbf{0} & \boldsymbol{K}_{\mathbf{0}} \\
\mathbf{0} & \boldsymbol{K}
\end{array}\right]\right)
$$

as the vector $\underline{\delta}_{0} \boldsymbol{K}_{\mathbf{0}}$ describes the probabilities of the call surviving the first handover in the different phases and the vector $\underline{\delta}_{0} K_{0} K^{i-1}$ describes the probabilities of the call surviving $i$ handovers in the different phases of the underlying Markov chain for the call-holding time. The scalar product with $\underline{e}$ then gives the sum of the probabilities for all the phases.

The probability of exactly $i$ handovers or visiting $i+1$ cells for $i \geq 1$ is given by

$$
p_{i}=P\{N=i\}=\underline{\delta}_{0} \boldsymbol{K}_{\mathbf{0}} \boldsymbol{K}^{i-1} \underline{K}^{0} .
$$

Here, $\underline{K}^{0}=(\underline{I}-\underline{K}) \underline{e}$ is the vector of absorption probabilities for the distribution of the number of handovers. The average number of handovers is given by

$$
E\{N\}=\sum_{i=0}^{\infty} P\{N>i\}=\sum_{i=0}^{\infty} q_{i+1}=\sum_{i=1}^{\infty} \underline{\delta}_{i} \cdot \underline{e} .
$$

Then the average number of cells visited is the average number of handovers plus one:

$$
\begin{aligned}
\sum_{i=1}^{\infty} \underline{\delta}_{i} \cdot \underline{e}+1 & =\sum_{i=1}^{\infty} \underline{\delta}_{0} \boldsymbol{K}_{\mathbf{0}} \boldsymbol{K}^{i-1} \underline{e}+1 \\
& =\underline{\delta}_{0} \boldsymbol{K}_{\mathbf{0}}(\boldsymbol{I}-\boldsymbol{K})^{-1} \underline{e}+1
\end{aligned}
$$

A more direct way to derive this result would be to use the mean of the discrete phase-type distribution. Since the probability that the call terminates in any given cell is $\left(\sum_{i=0}^{\infty} \underline{\delta}_{i} \underline{e}\right)^{-1}$, the fraction of ongoing channel occupancies in the $i$ th cell is given by

$$
\frac{\underline{\delta}_{i} \cdot \underline{e}}{\sum_{i=0}^{\infty} \underline{\delta}_{i} \cdot \underline{e}}=\frac{\underline{\delta}_{0} \boldsymbol{K}_{\mathbf{0}} \boldsymbol{K}^{i-1} \underline{\underline{e}}}{\underline{\delta}_{0} \boldsymbol{K}_{\mathbf{0}}(\boldsymbol{I}-\boldsymbol{K})^{-1} \underline{e}+1}, \quad i \geq 1
$$

The fraction of channel occupancies terminating in the $i$ th cell is

$$
\begin{aligned}
\frac{\underline{\delta}_{i-1} \cdot \underline{e}-\underline{\delta}_{i} \cdot \underline{e}}{\sum_{i=0}^{\infty} \underline{\delta}_{i} \cdot \underline{e}} & =\frac{\underline{\delta}_{0} \boldsymbol{K}_{\mathbf{0}} \boldsymbol{K}^{i-2} \underline{e}-\underline{\delta}_{0} \boldsymbol{K}_{\mathbf{0}} \boldsymbol{K}^{i-1} \underline{e}}{\underline{\delta}_{0} \boldsymbol{K}_{\mathbf{0}}(\boldsymbol{I}-\boldsymbol{K})^{-1} \underline{e}+1} \\
& =\frac{\underline{\delta}_{0} \boldsymbol{K}_{\mathbf{0}} \boldsymbol{K}^{i-2} \underline{K}^{0}}{\underline{\delta}_{0} \boldsymbol{K}_{\mathbf{0}}(\boldsymbol{I}-\boldsymbol{K})^{-1} \underline{e}+1}, \quad i \geq 2 .
\end{aligned}
$$

The fraction of channel occupancies in the first cell is

$$
\frac{1-\underline{\delta}_{1} \underline{e}}{\sum_{i=0}^{\infty} \underline{\delta}_{i} \cdot \underline{e}}=\frac{1-\underline{\delta}_{1} \underline{e}}{\underline{\delta}_{0} \boldsymbol{K}_{\mathbf{0}}(\boldsymbol{I}-\boldsymbol{K})^{-1} \underline{e}+1}
$$

The fractions of channel occupancies after the first cell by handover from the respective phases are given by the vector

$$
\frac{\sum_{i=1}^{\infty} \underline{\delta}_{i}}{\sum_{i=0}^{\infty} \underline{\delta}_{i} \cdot \underline{e}}=\frac{\underline{\delta}_{0} \boldsymbol{K}_{\mathbf{0}}(\boldsymbol{I}-\boldsymbol{K})^{-1}}{\underline{\delta}_{0} \boldsymbol{K}_{\mathbf{0}}(\boldsymbol{I}-\boldsymbol{K})^{-1} \underline{e}+1}
$$

\section{Channel-Holding Time in a Cell}

Define $Z$ as the channel occupancy or channel-holding time in a cell. A channel occupancy is caused by a new call with probability $\underline{\delta}_{0} \underline{e} / \sum_{i=0}^{\infty} \underline{\delta}_{i} \underline{e}$, by a handover with probability $\sum_{i=1}^{\infty} \underline{\delta}_{i} \underline{e} / \sum_{i=0}^{\infty} \underline{\delta}_{i} \underline{e}$, or from the different phases with probabilities $\underline{\delta}_{0} / \sum_{i=0}^{\infty} \underline{\delta}_{i} \underline{e}$ and $\sum_{i=1}^{\infty} \underline{\delta}_{i} / \sum_{i=0}^{\infty} \underline{\delta}_{i} \underline{e}$, respectively. Channel occupancy terminates either because the call has terminated or because it has been handed over to a new cell. This implies that the channel-holding time in a cell is the minimum of the cell-residence time and the remaining call time. The minimum of two phase-type distributions is phase-type distributed [10]. Thus, $Z \in P H(\underline{\gamma}, \boldsymbol{Q})$, where using (21)

$$
\begin{aligned}
& \underline{\gamma}=\left(\underline{\beta}_{0} \otimes \frac{\underline{\delta}_{0}}{\underline{\delta}_{0} \boldsymbol{K}_{\mathbf{0}}(\boldsymbol{I}-\boldsymbol{K})^{-1} \underline{e}+1},\right. \\
&\left.\underline{\beta} \otimes \frac{\underline{\delta}_{0} \boldsymbol{K}_{\mathbf{0}}(\boldsymbol{I}-\boldsymbol{K})^{-1}}{\underline{\delta}_{0} \boldsymbol{K}_{\mathbf{0}}(\boldsymbol{I}-\boldsymbol{K})^{-1} \underline{e}+1}\right) \\
& \boldsymbol{Q}=\left[\begin{array}{cc}
\boldsymbol{S}_{\mathbf{0}} \oplus \boldsymbol{T} & \mathbf{0} \\
\mathbf{0} & \boldsymbol{S} \oplus \boldsymbol{T}
\end{array}\right] .
\end{aligned}
$$

\section{E. Conditional Channel-Holding Times}

Absorption due to a handover occurs with the intensities $\underline{S}_{0}{ }^{0} \otimes \underline{e}$ in the first cell and $\underline{S}^{0} \otimes \underline{e}$ in any other cell and absorption due to a terminated call occurs with the intensities $\underline{e} \otimes \underline{T}^{0}$. The channel-holding time is now interpreted as the time to absorption in a Markov chain with two absorbing states: handover and terminated call. Thus, the channel-holding time conditioned on a handover and a terminated call, respectively, can be derived. Define $V$ to be the channel-holding time conditioned on absorption due to a handover and $W$ as the channel-holding time conditioned on absorption due to a terminated call.

Then, $V \in \operatorname{PH}\left(\underline{\gamma}_{V}, \boldsymbol{R}\right)$ and $W \in \operatorname{PH}\left(\underline{\gamma}_{W}, \boldsymbol{R}\right)[1] . \underline{\gamma}_{V}$ describes the transient states from which the absorption occurred, given the absorption was due to a handover, and $\underline{\gamma}_{W}$, from which 
the absorption of transient states occurred, given the absorption was due to a terminated call

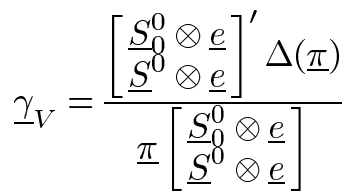

$$
\begin{aligned}
& \underline{\gamma}_{W}=\frac{\left(\underline{e} \otimes \underline{T}^{0}\right)^{\prime} \Delta(\underline{\pi})}{\underline{\pi}\left(\underline{e} \otimes \underline{T}^{0}\right)} \text {. }
\end{aligned}
$$

Here, $\Delta(\underline{\pi})$ is a diagonal matrix with diagonal elements from the stationary probability vector $\underline{\pi}$ of a PH renewal process that is given by

$$
\underline{\pi}=\frac{\underline{\gamma}(-Q)^{-1}}{\underline{\gamma}(-Q)^{-1} \underline{e}} .
$$

The generator matrix $R$ for the transient part is given by

$$
\boldsymbol{R}=\Delta^{-1}(\underline{\pi}) \boldsymbol{Q}^{\prime} \Delta(\underline{\pi}) \text {. }
$$

\section{F. Correlated Cell-Residence Times}

If we define a handover as a renewal, then the model can be thought of as a renewal process, with interval distribution of phase-type, observed in a phase-type distributed time interval, namely the call-holding time. Once the Markov chain related to the inter renewal times has evolved to absorption, it is instantaneously restarted by choosing a state according to the distribution $\beta$.

The generator matrix of the Markov chain is split in the matrices $\boldsymbol{S}$ and $\underline{T}^{0} \beta$. The first matrix is related to phase transitions without renewals - in this case, handovers - and the second matrix is related to phase transitions associated with renewals.

In the more general MAP, $D=D_{0}+D_{1}$ is an irreducible generator of a Markov chain. $D_{0}$ is associated with transitions without arrivals and $D_{\mathbf{1}}$ is associated with transitions due to arrivals. The phase-type renewal process is a MAP with $D_{0}=S$ and $\boldsymbol{D}_{\mathbf{1}}=\underline{S}^{0} \beta$ [10].

Assume that handovers occur according to an $m$ state MAP with generator matrix $\boldsymbol{D}_{\mathbf{0}}+\boldsymbol{D}_{\mathbf{1}}$; then, it is possible to have correlated cell-residence times. The call-holding time is $\operatorname{PH}\left(\underline{\delta}_{0}, \boldsymbol{T}\right)$ with $n$ states. By combining the states of the MAP-describing cell-residence times and the states of the call-holding time, a new Markov chain with a two-dimensional (2-D) state space is obtained, containing $n \cdot m$ states, denoted $(j, k)$, where $j$ is the state of the call-holding time and $k$ is the state of the cell-residence times. Define $\tilde{\delta}_{i, j, k}=P\left\{J\left(\tau_{i}\right)=(j, k)\right\}$, where $\tilde{\delta}_{i, j, k}$ is the probability that the Markov chain is in phase $j$ for the call-holding time and phase $k$ in the MAP-describing cell-residence times at the $i$ th handover. Fig. 2 is an illustration of the idea behind the initial model that also illustrates the approach for this model, the only difference being that $\underline{\tilde{\delta}}_{i}$ now also depends on the cell-residence time. Thus

$$
\underline{\tilde{\delta}}_{i}=\left[\tilde{\delta}_{i, 1,1}, \ldots, \tilde{\delta}_{i, 1, m}, \ldots, \tilde{\delta}_{i, n, 1}, \ldots, \tilde{\delta}_{i, n, m}\right]
$$

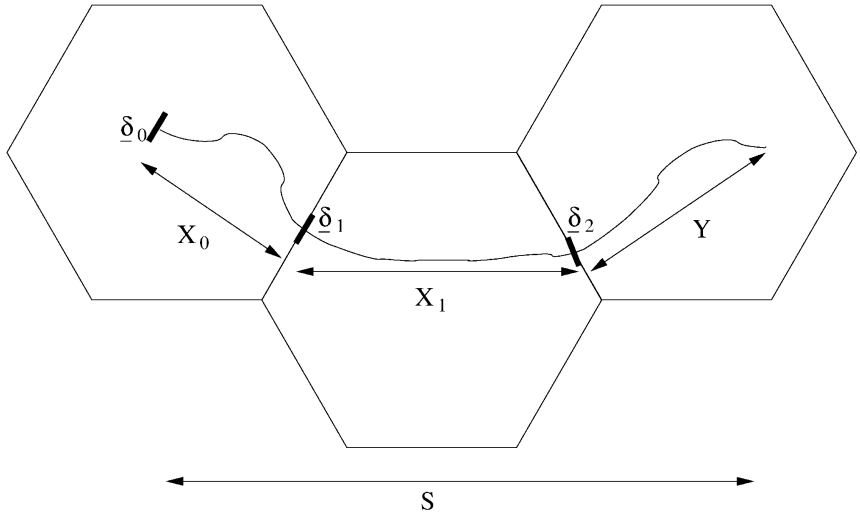

Fig. 2. Idea behind our approach is that the transition matrix of the channelholding time must keep track of the states of both the cell-residence times $\left(X_{0}, X_{i}\right.$, and $\left.Y\right)$ and the total call-holding time $(S)$. If either processes are absorbed, so is the process for the channel-holding time. With $\underline{\delta}_{i}$ being the joint probabilities of having experienced $i$ handovers, averaging over all $\underline{\delta}_{i}$ gives us the initial vector for the phase-type distribution of the channel-holding time.

With the initial distribution $\underline{\beta}$ for the MAP, the initial probability vector is

$$
\underline{\tilde{\delta}}_{0}=\underline{\delta}_{0} \otimes \underline{\beta} .
$$

With this definition of $\tilde{\delta}$, (3) becomes

$$
\begin{aligned}
\tilde{\tilde{\delta}}_{1} & =\underline{\tilde{\delta}}_{0} \cdot \int_{0}^{\infty} e^{\left(\boldsymbol{T} \oplus \boldsymbol{D}_{\mathbf{0}}\right) u}\left(\boldsymbol{I} \otimes \boldsymbol{D}_{\mathbf{1}}\right) d u \\
\tilde{\tilde{\delta}}_{i+1} & =\underline{\tilde{\delta}}_{i} \cdot \int_{0}^{\infty} e^{\left(\boldsymbol{T} \oplus \boldsymbol{D}_{\mathbf{0}}\right) u}\left(\boldsymbol{I} \otimes \boldsymbol{D}_{\mathbf{1}}\right) d u, \quad i \geq 1 .
\end{aligned}
$$

This gives

$$
\begin{aligned}
& \underline{\tilde{\delta}}_{i}=\underline{\tilde{\delta}}_{i-1} \boldsymbol{K}, \quad i \geq 1 \\
& \underline{\tilde{\delta}}_{i}=\underline{\tilde{\delta}}_{0} \boldsymbol{K}^{i}, \quad i \geq 1
\end{aligned}
$$

with

$$
\boldsymbol{K}=-\left(\boldsymbol{T} \oplus \boldsymbol{D}_{0}\right)^{-1}\left(\boldsymbol{I} \otimes \boldsymbol{D}_{\mathbf{1}}\right) .
$$

This method of deriving $\boldsymbol{K}_{\mathbf{0}}$ and $\boldsymbol{K}$ could have been used for the model with uncorrelated cell-residence times; however, to do this it would be necessary to construct a product space for the cell-residence and call-holding times, increasing the size of the state space. Note that (11)-(19) still hold with $\boldsymbol{K}_{\mathbf{0}}=\boldsymbol{K}$. The channel-holding time is now $Z \in P H(\underline{\gamma}, \boldsymbol{Q})$ where the initial probability vector is

$$
\underline{\gamma}=\frac{\tilde{\tilde{\delta}}_{0}+\underline{\tilde{\delta}}_{0} \boldsymbol{K}(\boldsymbol{I}-\boldsymbol{K})^{-1}}{\underline{\tilde{\delta}}_{0} \boldsymbol{K}(\boldsymbol{I}-\boldsymbol{K})^{-1} \underline{e}+1}=\frac{\tilde{\tilde{\delta}}_{0}(\boldsymbol{I}-\boldsymbol{K})^{-1}}{\underline{\tilde{\delta}}_{0}(\boldsymbol{I}-\boldsymbol{K})^{-1} \underline{e}} .
$$

The transition matrix is

$$
Q=T \oplus D_{0}
$$

and the absorption rates are

$$
\underline{Q}^{0}=\underline{T}^{0} \oplus \boldsymbol{D}_{\mathbf{1}} \underline{e} .
$$



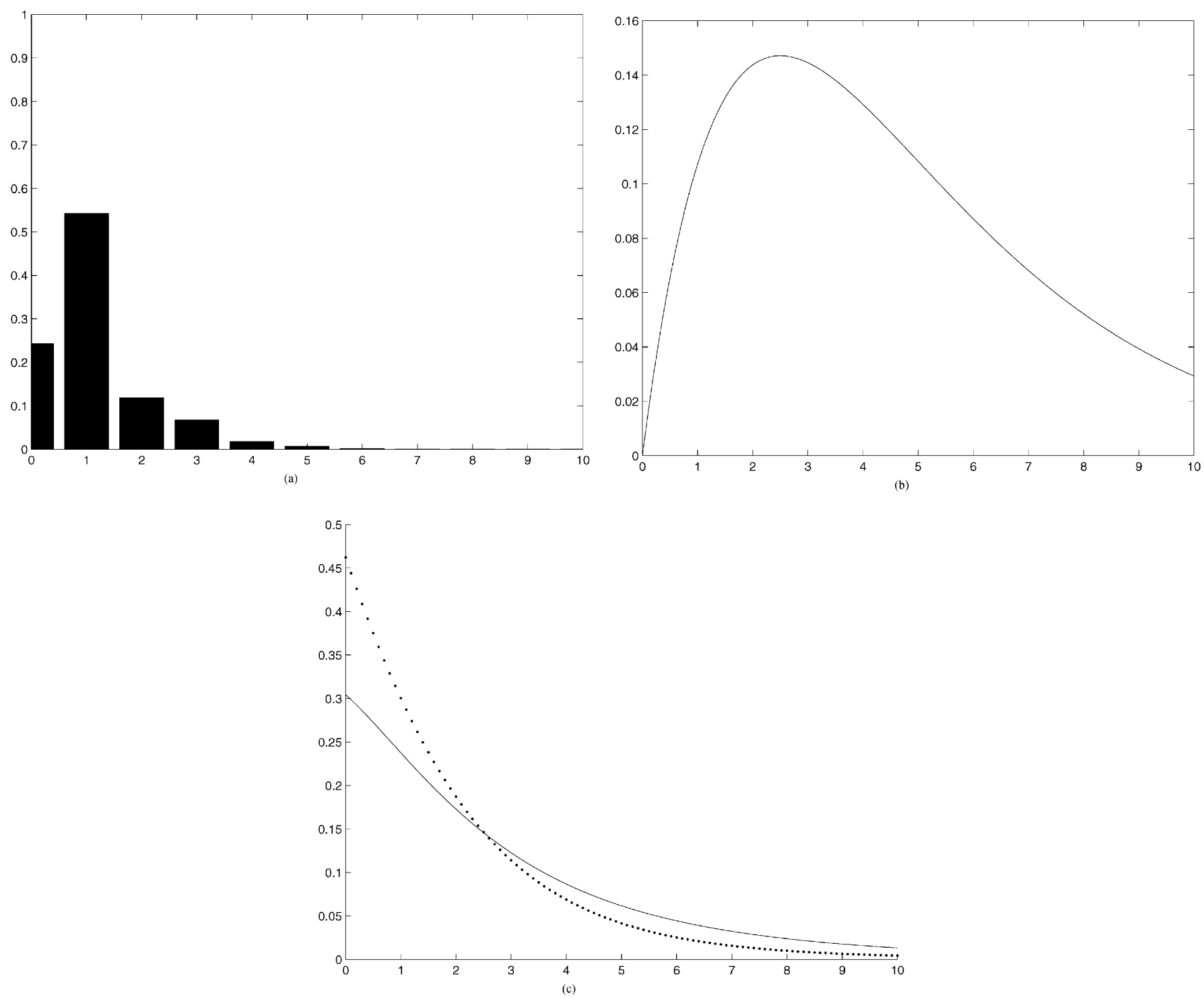

Fig. 3. (a) Distribution of handovers for the correlated instance, (b) call-holding and (c) channel-holding times for the correlated instance (solid line) and the uncorrelated instance (dotted line).

\section{CASE STUDY}

Let cell changes occur according to a MAP and let the callholding time be Erlang-2 distributed with intensity $\lambda_{3}$ in both phases as

$$
\begin{aligned}
X: \underline{\beta}=(q, 1-q) & \boldsymbol{D}_{0}=\left[\begin{array}{cc}
-\lambda_{1} & 0 \\
0 & -\lambda_{2}
\end{array}\right] \\
\boldsymbol{D}_{\mathbf{1}} & =\left[\begin{array}{cc}
\lambda_{1}\left(1-\epsilon_{1}\right) & \lambda_{1} \epsilon_{1} \\
\lambda_{2} \epsilon_{2} & \lambda_{2}\left(1-\epsilon_{2}\right)
\end{array}\right] \\
S: \underline{\delta}_{0}=(1,0) & \boldsymbol{T}=\left[\begin{array}{cc}
-\lambda_{3} & \lambda_{3} \\
0 & -\lambda_{3}
\end{array}\right] .
\end{aligned}
$$

Fig. 3 shows one possible realization of this model with $q=$ $0.1, \epsilon_{1}=0.85, \epsilon_{2}=0.85, \lambda_{1}=0.04, \lambda_{2}=0.4$, and $\lambda_{3}=0.3$. The cell-residence time can be in two different states, one where handovers occur very infrequently and one where handovers occur frequently. However, the cell-residence times are correlated meaning that if handovers occur frequently, they are likely to continue doing so. An example of this could be calls initiated on a highway, where there is heavy traffic during rush hour.
The transition matrix between handovers is then

$$
\boldsymbol{K}=\left[\begin{array}{cccc}
0.0176 & 0.1000 & 0.0156 & 0.0882 \\
0.4857 & 0.0857 & 0.2082 & 0.0367 \\
0 & 0 & 0.0176 & 0.1000 \\
0 & 0 & 0.4857 & 0.0857
\end{array}\right]
$$

The mean number of handovers is 1.1094 and the channelholding time is $P H(\underline{\gamma}, \boldsymbol{Q})$ with

$$
\begin{aligned}
& \underline{\gamma}=(0.2950,0.4989,0.1421,0.0640) \\
& \boldsymbol{Q}=\left[\begin{array}{cccc}
-0.34 & 0 & 0.30 & 0 \\
0 & -0.70 & 0 & 0.30 \\
0 & 0 & -0.34 & 0 \\
0 & 0 & 0 & -0.70
\end{array}\right]
\end{aligned}
$$

In Fig. 3(c), the effect of the correlation is evident. This illustrates how the fact that channel-holding times in general are nonexponential may also be due in part to correlation between cell-residence times. A more direct choice of parameters is to choose $\epsilon_{1}=\epsilon_{2}=0$. One sees the same overall effect 

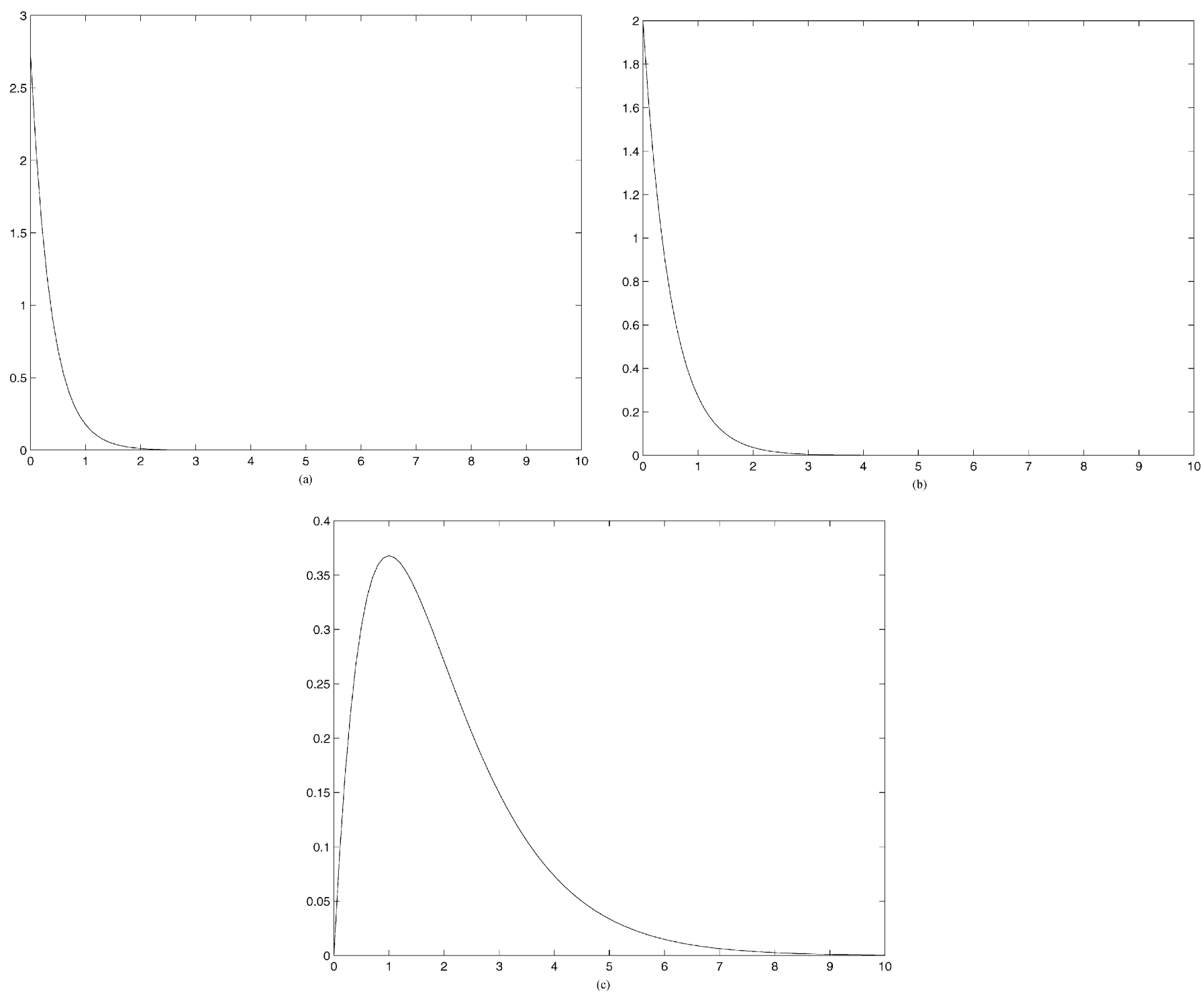

Fig. 4. (a) Cell-residence time in the first cell. (b) Cell-residence time in the following cells. (c) Total call-holding time.

\section{CONCLUSION}

This paper presents a statistically based approach to the problem of modeling channel-holding times and provides a unified framework including the Cox, [15], SOHYP, and hyper-Erlang models, proposed in [5] and [6]. The channelholding time distribution has been derived for phase-type distributed call-holding and cell-residence times, in the case of uncorrelated and correlated cell-residence times, both cases resulting in a distribution of phase-type. Some of the results may be generalized to general distributions. Although our approach might seem complicated, if one is not used to matrix formalism, formulas are easy to implement and generally are numerically stable. Since phase-type distributions provide approximations for general distributions, we consider the results presented in this paper to be a big step in the line of development toward a general and tractable modeling tool for channel-holding times.

In addition to the channel-holding time distribution, the distribution of the number of cells visited and the channel-holding times conditioned on the call being a hand-off call or a new call have also been derived.

The results are illustrated by a simple case with cell changes occurring according to a simple MAP and Erlang-2 distributed call-holding times. Estimation in phase-type models is a hot research topic with results available for the practitioner [8]. For a recent survey of MAP estimation procedures, see [14].

\section{APPENDIX \\ ANALYTICAL EXAMPLE}

Let the residence time in the first cell $X_{0}$ and in the $i$ th cell $X_{i}$ be exponentially distributed with intensities $\lambda_{1}$ and $\lambda_{2}$, respectively, and let the call-holding time be Erlang-2 distributed with intensity $\lambda_{3}$ as

$$
\begin{aligned}
X_{0}: \underline{\beta}_{0} & =1 \quad S_{0}=-\lambda_{1} \\
X_{i}: \underline{\beta} & =1 \quad \boldsymbol{S}=-\lambda_{2} \\
S: \underline{\delta}_{0} & =(1,0) \quad \boldsymbol{T}=\left[\begin{array}{cc}
-\lambda_{3} & \lambda_{3} \\
0 & -\lambda_{3}
\end{array}\right] .
\end{aligned}
$$


An illustration of the distribution function for these can be found in Fig. 4.

$$
\begin{aligned}
& \theta=\max \left\{\left|T_{i i}\right|: 1 \leq i \leq n\right\}=\lambda_{3} \\
& \boldsymbol{A}=\boldsymbol{I}+\theta^{-1} \boldsymbol{T}=\left[\begin{array}{ll}
0 & 1 \\
0 & 0
\end{array}\right] .
\end{aligned}
$$

The transition matrices $K_{0}$ and $K$ are then given by

$$
\begin{aligned}
\boldsymbol{K}_{\mathbf{0}} & =\left[\begin{array}{cc}
\frac{\lambda_{1}}{\lambda_{1}+\lambda_{3}} & \frac{\lambda_{1} \lambda_{3}}{\left(\lambda_{1}+\lambda_{3}\right)^{2}} \\
0 & \frac{\lambda_{1}}{\lambda_{1}+\lambda_{3}}
\end{array}\right] \\
\boldsymbol{K} & =\left[\begin{array}{cc}
\frac{\lambda_{2}}{\lambda_{2}+\lambda_{3}} & \frac{\lambda_{2} \lambda_{3}}{\left(\lambda_{2}+\lambda_{3}\right)^{2}} \\
0 & \frac{\lambda_{2}}{\lambda_{2}+\lambda_{3}}
\end{array}\right] .
\end{aligned}
$$

The probability that the call terminates in the first cell is given by

$$
p_{0}=\left(\frac{\lambda_{3}}{\lambda_{1}+\lambda_{3}}\right)^{2} .
$$

The probability that the call survives $i$ cells is given by

$$
\begin{aligned}
q_{i}=\frac{\lambda_{1}}{\lambda_{1}+\lambda_{3}} & \left(\frac{\lambda_{2}}{\lambda_{2}+\lambda_{3}}\right)^{i-1} \\
& \times\left(1+\frac{(i-1) \lambda_{3}}{\lambda_{2}+\lambda_{3}}+\frac{\lambda_{3}}{\lambda_{1}+\lambda_{3}}\right), \quad i \geq 1 .
\end{aligned}
$$

The distribution of the number of handovers can be seen in Fig. 5. The average number of cells visited is

$$
E(N+1)=\frac{f\left(\lambda_{1}, \lambda_{2}, \lambda_{3}\right)}{\lambda_{3}\left(\lambda_{1}+\lambda_{3}\right)^{2}}
$$

where $f\left(\lambda_{1}, \lambda_{2}, \lambda_{3}\right)=\lambda_{1}\left(\lambda_{2}+\lambda_{3}\right)\left(\lambda_{1}+\lambda_{3}\right)+\lambda_{1} \lambda_{2}\left(\lambda_{1}+\right.$ $\left.\lambda_{3}\right)+\lambda_{1} \lambda_{3}\left(\lambda_{2}+\lambda_{3}\right)+\lambda_{3}\left(\lambda_{1}+\lambda_{3}\right)^{2}$. The following calculation is necessary for the determination of $\gamma$ :

$$
\sum_{i=1}^{\infty} \underline{\delta}_{i}=\frac{\lambda_{1}}{\lambda_{3}} \frac{\lambda_{2}+\lambda_{3}}{\lambda_{1}+\lambda_{3}}\left(1, \frac{\lambda_{2}\left(\lambda_{1}+\lambda_{3}\right)+\lambda_{3}\left(\lambda_{2}+\lambda_{3}\right)}{\left(\lambda_{1}+\lambda_{3}\right)\left(\lambda_{2}+\lambda_{3}\right)}\right) .
$$

The channel-holding time in the cells is phase-type distributed $\operatorname{PH}(\underline{\gamma}, \boldsymbol{Q})$, where $\underline{\gamma}$ is

$$
\underline{\gamma}=\left(\underline{\beta}_{0} \otimes \frac{\underline{\delta}_{0}}{\sum_{i=0}^{\infty} \underline{\delta}_{i} \underline{e}}, \underline{\beta} \otimes \frac{\sum_{i=1}^{\infty} \underline{\delta}_{i}}{\sum_{i=0}^{\infty} \underline{\delta}_{i} \underline{e}}\right)=\left(\gamma_{1}, \gamma_{2}, \gamma_{3}, \gamma_{4}\right)
$$

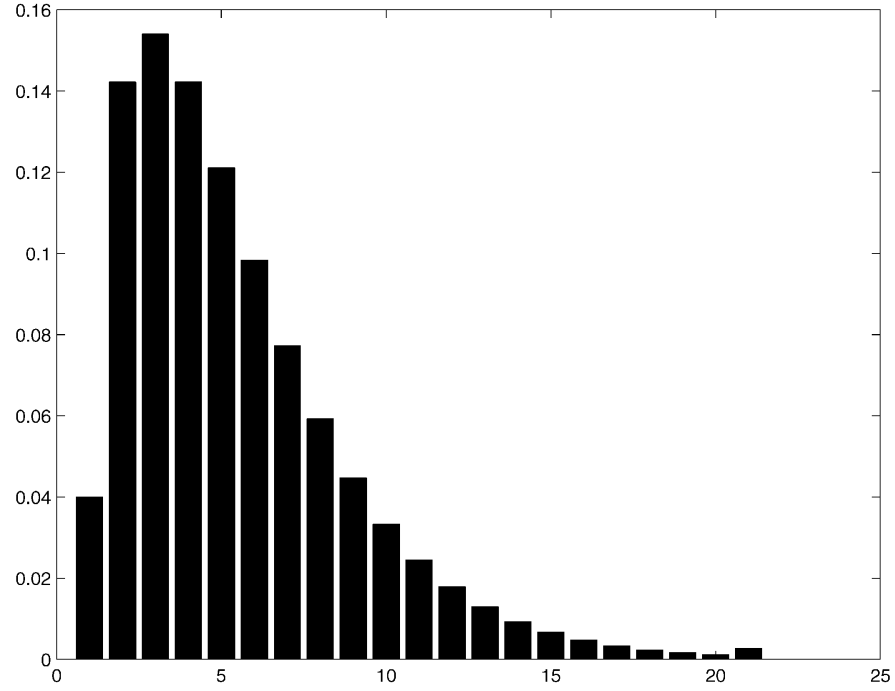

Fig. 5. The distribution of the number of handovers.

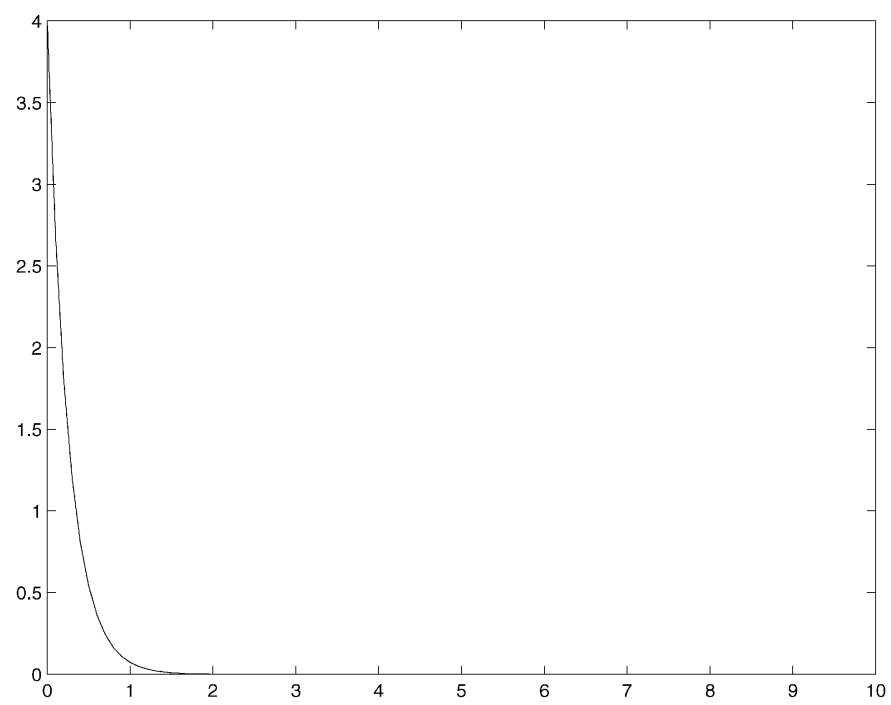

Fig. 6. The distribution of the channel-holding time.

with

$$
\begin{aligned}
\gamma_{1} & =\frac{\lambda_{3}\left(\lambda_{1}+\lambda_{3}\right)^{2}}{f\left(\lambda_{1}, \lambda_{2}, \lambda_{3}\right)} \\
\gamma_{2} & =0 \\
\gamma_{3} & =\frac{\lambda_{1}\left(\lambda_{1}+\lambda_{3}\right)\left(\lambda_{2}+\lambda_{3}\right)}{f\left(\lambda_{1}, \lambda_{2}, \lambda_{3}\right)} \\
\gamma_{4} & =\frac{\lambda_{1} \lambda_{2}\left(\lambda_{1}+\lambda_{3}\right)+\lambda_{1} \lambda_{3}\left(\lambda_{2}+\lambda_{3}\right)}{f\left(\lambda_{1}, \lambda_{2}, \lambda_{3}\right)}
\end{aligned}
$$

and $f\left(\lambda_{1}, \lambda_{2}, \lambda_{3}\right)$ is the same as above. $\boldsymbol{Q}$ is given by the equation at the bottom of the page. Fig. 6 shows the resulting distribu-

$$
\begin{aligned}
Q & =\left[\begin{array}{cc}
\boldsymbol{S}_{\mathbf{0}} \oplus \boldsymbol{T} & \mathbf{0} \\
\mathbf{0} & \boldsymbol{S} \oplus \boldsymbol{T}
\end{array}\right] \\
& =\left[\begin{array}{cccc}
-\left(\lambda_{1}+\lambda_{3}\right) & \lambda_{3} & 0 & 0 \\
0 & -\left(\lambda_{1}+\lambda_{3}\right) & 0 & 0 \\
0 & 0 & -\left(\lambda_{2}+\lambda_{3}\right) & \lambda_{3} \\
0 & 0 & 0 & -\left(\lambda_{2}+\lambda_{3}\right)
\end{array}\right] .
\end{aligned}
$$




$$
\boldsymbol{R}=\left[\begin{array}{cccc}
-\left(\lambda_{1}+\lambda_{3}\right) & 0 & 0 & 0 \\
\lambda_{1}+\lambda_{3} & -\left(\lambda_{1}+\lambda_{3}\right) & 0 & 0 \\
0 & 0 & -\left(\lambda_{2}+\lambda_{3}\right) & 0 \\
0 & 0 & \frac{\lambda_{3}\left(\lambda_{1}+\lambda_{3}\right)}{\lambda_{1}+2 \lambda_{3}} & -\left(\lambda_{2}+\lambda_{3}\right)
\end{array}\right]
$$

tion function for the channel-holding time. The channel-holding time conditioned on absorption due to a handover is $\mathrm{PH}\left(\underline{\gamma}_{V}, \boldsymbol{R}\right)$ and the channel-holding time conditioned on absorption due to a terminated call is $\mathrm{PH}\left(\underline{\gamma}_{W}, \boldsymbol{R}\right)$ where

$$
\begin{aligned}
\underline{\gamma}_{V} & =\left(\gamma_{V 1}, \gamma_{V 2}, \gamma_{V 3}, \gamma_{V 4}\right) \\
\underline{\gamma}_{W} & =\left(\gamma_{W 1}, \gamma_{W 2}, \gamma_{W 3}, \gamma_{W 4}\right)
\end{aligned}
$$

with

$$
\begin{aligned}
\gamma_{V 1} & =\frac{\lambda_{3}\left(\lambda_{1}+\lambda_{3}\right)}{\lambda_{1} \lambda_{3}+2 \lambda_{1} \lambda_{2}+3 \lambda_{2} \lambda_{3}+2 \lambda_{3}^{2}} \\
\gamma_{V 2} & =\frac{\lambda_{3}^{2}}{\lambda_{1} \lambda_{3}+2 \lambda_{1} \lambda_{2}+3 \lambda_{2} \lambda_{3}+2 \lambda_{3}^{2}} \\
\gamma_{V 3} & =\frac{\lambda_{2}\left(\lambda_{1}+\lambda_{3}\right)}{\lambda_{1} \lambda_{3}+2 \lambda_{1} \lambda_{2}+3 \lambda_{2} \lambda_{3}+2 \lambda_{3}^{2}} \\
\gamma_{V 4} & =\frac{\lambda_{2}\left(\lambda_{1}+2 \lambda_{3}\right)}{\lambda_{1} \lambda_{3}+2 \lambda_{1} \lambda_{2}+3 \lambda_{2} \lambda_{3}+2 \lambda_{3}^{2}} \\
\gamma_{W 1} & =\frac{\lambda_{3}}{2 \lambda_{1}+2 \lambda_{3}} \\
\gamma_{W 2} & =\frac{\lambda_{3}^{2}}{4 \lambda_{1} \lambda_{3}+2 \lambda_{1}^{2}+2 \lambda_{3}^{2}} \\
\gamma_{W 3} & =\frac{\lambda_{1}}{2 \lambda_{1}+2 \lambda_{3}} \\
\gamma_{W 4} & =\frac{\lambda_{1}\left(\lambda_{1}+2 \lambda_{3}\right)}{4 \lambda_{1} \lambda_{3}+2 \lambda_{1}^{2}+2 \lambda_{3}^{2}}
\end{aligned}
$$

and (see equation at the top of the page).

\section{REFERENCES}

[1] A. T. Andersen, M. F. Neuts, and B. F. Nielsen, " $P H$-Distributions arising through conditioning," Commun. Stat.-Stochastic Models, vol. 16, no. 1, pp. 179-188, 2000.

[2] F. Barceló and J. Jordán, "Channel holding time distribution in public cellular telephony," in Proc. 16th Int. Teletraffic Cong., Edinburgh, U.K., June 1999, pp. 107-116.

[3] E. Chlebus, T. Zbiezek, and W. Ludwin, "Analysis of channel holding time in wireless mobile systems: Does the probability distribution of cell residence time matter?," in Proc. 16th Int. Teletraffic Cong., Edinburgh, U.K., June 1999, pp. 117-128.

[4] T. K. Christensen, "Integration of Voice and Data in GPRS," Master, Dept. Informatics and Mathematical Modeling, Tech. Univ. Denmark, Lyngby, 2001.

[5] Y. Fang and I. Chlamtac, "Teletrafic analysis and mobility modeling of PCS networks," IEEE Trans. Commun., vol. 47, pp. 1062-1072, July 1999.

[6] Y. Fang, "Hyper-Erlang distribution model and its applications in wireless mobile networks," Wireless Networks, vol. 7, pp. 211-219, 2001.

[7] R. A. Guérin, "Channel occupancy time distribution in a cellular radio system," IEEE Trans. Veh. Technol., vol. VT-35, pp. 89-99, Feb. 1987.

[8] O. Häggström, S. Asmussen, and O. Nerman, "Fitting phase-type distributions via the EM algorithm," Scand. J. Stat., vol. 23, pp. 419-441, 1996.

[9] V. B. Iversen, "A generalization of the classical teletraffic theory," in Proc. Eleventh Int. Teletraffic Cong., Kyoto, Japan, 1985, pp. 58-64.

[10] G. Latouche and V. Ramaswami, Introduction to Matrix Analytic Methods in Stochastic Modeling. Philadelphia, PA: SIAM, 1999.
[11] R. Litjens and R. J. Boucherie, "Radio resource sharing in a GSM/GPRS network," in Proc. ITC Specialist Seminar Mobile Systems and Mobility, 2000, pp. 261-274.

[12] P. V. Orlik and S. S. Rappaport, "A model for teletraffic performance and channel holding time characterization in wireless cellular communication with general session and dwell time distributions," IEEE J. Select. Areas Commun., vol. 16, pp. 788-803, June 1998.

[13] M. Rajaratnam and F. Takawira, "Nonclassical traffic modeling and performance analysis of cellular mobile networks with and without channel reservation," IEEE Trans. Veh. Technol., vol. 49, pp. 817-834, May 2000.

[14] T. Rydén, "Statistical estimation for Markov-modulated Poisson processes and Markovian arrival processes," in Proc. Third Int. Conf. Matrix-Analytical Methods in Stochastic Models, Leuven, Belgium, July 2000, pp. 329-350.

[15] B. H. Soong and J. A. Barria, "A Coxian model for channel holding time distribution for teletraffic mobility modeling," IEEE Commun. Lett., vol. 4, pp. 402-404, Sept. 2000.

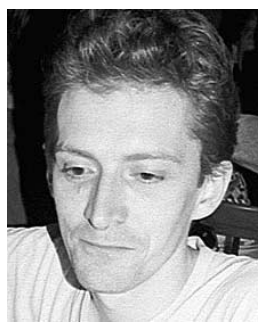

Thomas Kaare Christensen (S'01) received the M.Sc. degree in informatics from The Technical University of Denmark, Lyngby, in 2001. He was a Graduate Student in the Informatics and Mathematical Modeling Department, The Technical University of Denmark, until August 2003, when he passed away after a sudden detoriation in his condition caused by an acute disease. This paper was written as a part of his studies.

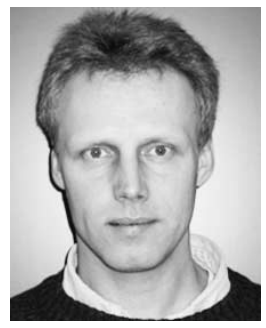

Bo Friis Nielsen (M'84) received the M.Sc. degree in electrical engineering and the Ph.D. degree in operations research from The Technical University of Denmark, Lyngby, in 1984 and 1988, respectively.

$\mathrm{He}$ is a Lecturer in the Department of Mathematical Modeling, The Technical University of Denmark. He has authored and coauthored a number of papers in journals and conference proceedings. His current research interests include characterization of point processes, the Markovian arrival process, phase-type distributions, and applications of these models in science and engineering.

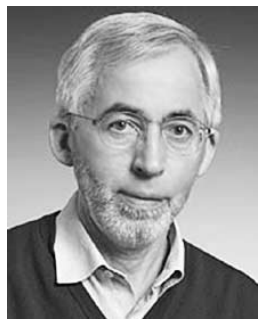

Villy Bæk Iversen (M'88) was born in Denmark in 1942. He received the M.Sc. degree in electrical engineering in 1968 and the Ph.D. degree in teletraffic engineering in 1976, both from The Technical University of Denmark, Lyngby.

$\mathrm{He}$ is an Associate Professor in the Department of Telecommunications, The Technical University of Denmark, where he teaches teletraffic engineering. In 1973, he was a Researcher at University of Stuttgart, Germany, and has visited many developing countries as an expert for the International Telecommunication Union. He has published about 90 papers and has edited several conference proceedings. His research interests include stochastic modeling, communication systems, and teletraffic engineering.

Dr. Iversen is Professor Honoris Causa at Beijing University of Posts and Telecommunications, Beijing, China, Vice Chairman of the International Advisory Committee of the International Teletraffic Congresses, and a Danish Member of IFIP TC6. 